\title{
Halloysite Nanotubes Noncovalently Functionalised with SDS Anionic Surfactant and PS-b-P4VP Block Copolymer for Their Effective Dispersion in Polystyrene as UV-Blocking Nanocomposite Films
}

\author{
Lazaros Tzounis, ${ }^{1,2}$ Shreya Herlekar, ${ }^{1,3}$ Antonios Tzounis, ${ }^{4}$ Nikolaos D. Charisiou, ${ }^{5}$ \\ Maria Goula, ${ }^{5}$ and Manfred Stamm ${ }^{1}$ \\ ${ }^{1}$ Leibniz-Institut für Polymerforschung Dresden, Hohe Straße 6, 01069 Dresden, Germany \\ ${ }^{2}$ Department of Materials Science and Engineering, University of Ioannina, 45110 Ioannina, Greece \\ ${ }^{3}$ Indian Institute of Technology, Kharagpur, India \\ ${ }^{4}$ Department of Agriculture, Crop Production \& Rural Environment, University of Thessaly, Nea Ionia, Volos, 38446 Magnesia, Greece \\ ${ }^{5}$ Laboratory of Alternative Fuels and Environmental Catalysis, Department of Environmental and Pollution Control Engineering, \\ School of Applied Sciences, Technological Education Institution of Western Macedonia (TEIWM), 50100, Koila, Kozani, Greece
}

Correspondence should be addressed to Lazaros Tzounis; ltzounis@cc.uoi.gr

Received 26 October 2016; Revised 21 January 2017; Accepted 9 February 2017; Published 19 March 2017

Academic Editor: Jim Low

Copyright (C) 2017 Lazaros Tzounis et al. This is an open access article distributed under the Creative Commons Attribution License, which permits unrestricted use, distribution, and reproduction in any medium, provided the original work is properly cited.

A simple and versatile method is reported for the noncovalent functionalisation of natural and "green" halloysite nanotubes (HNTs) allowing their effective dispersion in a polystyrene (PS) thermoplastic matrix via solvent mixing. Initially, HNTs (pristine HNTs) were modified with physically adsorbed surfactant molecules of sodium dodecyl sulphate (SDS) and PS-b-P4VP [P4VP: poly(4vinylpyridine)] block copolymer (BCP). Hereafter, SDS and BCP modified HNTs will be indicated as SDS-m-HNT and BCP-mHNT. Nanocomposite films with 1, 2, and $5 \mathrm{wt}$ \% HNT loadings were prepared, abbreviated as PS-SDS-m-HNT1, PS-SDS-m-HNT2, and PS-SDS-m-HNT5 and PS-BCP-m-HNT1, PS-BCP-m-HNT2, and PS-BCP-m-HNT5 (where 1, 2, and 5 correspond to the wt.\% of HNTs). All nanocomposites depicted improved thermal degradation compared to the neat PS as revealed by thermogravimetric analysis (TGA). Transmission electron microscopy (TEM) confirmed the good dispersion state of HNTs and the importance of modification by SDS and BCP. X-ray diffraction (XRD) studies showed the characteristic interlayer spacing between the two silicate layers of pristine and modified HNTs. The PS/HNT nanocomposite films exhibited excellent ultraviolent-visible (UV-vis) absorbance properties and their potential application as UV-filters could be envisaged.

\section{Introduction}

In the past decades, polymer nanocomposites reinforced with different kinds of nanoscale materials such as natural mineral layered silicate clays, carbon nanotubes (CNTs), and graphite nanoplatelets have attracted a steadily growing scientific interest due to their peculiar and fascinating properties, as well as their unique applications in commercial sectors [19]. The properties of the resulting nanocomposites depend greatly on the physical and chemical properties of the polymer matrices, the nature and surface chemistry of nanofillers, and the method of preparation. In all cases, the uniform and homogeneous dispersion of nanofillers in the polymer matrixes, overcoming the tendency of nanoparticles to form agglomerates, is a general prerequisite for achieving desired mechanical and physical characteristics [10-13].

Naturally occurring clay-based polymer nanocomposites have attracted considerable attention owing to their significantly improved mechanical and thermal properties compared to conventional composites, even at low clay loadings [14-20]. The clay minerals investigated are principally lamellar clays and most notably laponite [21, 22], 
smectite [23], kaolinite [24], and montmorillonite [25]. A natural clay material named as halloysite [26, 27] and its application in composites are still significantly unexplored. HNTs are naturally occurring two-layered aluminosilicate $\left[\mathrm{Al}_{2}(\mathrm{OH}) 4 \mathrm{Si}_{2} \mathrm{O}_{5}-2 \mathrm{H}_{2} \mathrm{O}\right]$ with a predominantly hollow tubular structure, with sizes ranging in the submicron range, chemically similar to kaolin, mined from natural deposits in countries such as America, China, New Zealand, France, and Belgium [28]. Mismatch in the two-layered alignment of the tetrahedral sheet of silica bonded to the octahedral or gibbsite sheet of alumina causes the wall to curve into the cylindrical shape as explained by Bates et al. [29]. Intercalated water may occur between the repetitive two-layered sheets comprising the spiral wall, which tends to be irreversibly removed on drying [28]. Halloysite is a low cost alternative green material that can be mined from the consequent deposit as a raw mineral. The size of halloysite particles varies between 1 and $15 \mu \mathrm{m}$ in length and 10 and $150 \mathrm{~nm}$ in diameter, depending on the deposits. Due to their high aspect ratio, HNTs give a large amount of filler-polymer interactions compared to other nanofillers. Moreover, the large diameter of the halloysite lumen is expected to accommodate some polymer molecules, which can offer polymeric composites with some specific properties like high degradation temperature and so forth [28]. Therefore, HNTs have been used as a new type of filler for polymers, that is, epoxies, polypropylene, polyvinyl alcohol, and so on, to improve the mechanical and thermal properties of the composites [30-33]. It has been shown also that polymer/HNT nanocomposites exhibited significantly reduced gas permeability, increased thermal stability, and improved flame retardancy [28, 30, 34, 35].

Similar to other clay materials, HNTs exhibit a large amount of surface hydroxyl groups rendering their dispersion into a polymer matrix and mostly to nonpolar ones very difficult $[28,36]$. This phenomenon is very familiar with well-established silica filler. Bifunctional organosilanes, such as bis(triethoxysilylpropyl)-tetrasulfide (TESPT), have been proposed to chemically modify silica surfaces for that purpose [30]. Yuan et al. [37] reported the modification of HNT by amino silane (3-aminopropyl) trimethoxysilane (APTES) and tried to understand the reaction chemistry behind it. Silane bearing glycidyl group treated HNTs were incorporated to a cyanate ester cured epoxy resin and have been found to enhance the dispersion of HNTs [38]. Most of the work published until now is related to the functionalisation of HNTs and it is limited to the mechanical reinforcement of polymer matrices. The noncovalent functionalisation of HNTs and their dispersion in a PS thermoplastic matrix for the production of nanocomposites with exceptional UV absorbance and blocking properties are yet to be explored.

Herein, a facile approach is presented for the noncovalent modification of HNTs and the preparation of PS/HNT nanocomposites with improved dispersion and exceptional UV absorbance properties. The improvement in the degree of dispersion of HNTs in the PS matrix is the main objective of the present work because the dispersion of HNTs in polymers is a difficult issue due to their hydrophilic nature. The first modification is achieved using the SDS anionic surfactant and the second using a BCP of the polystyrene-block-poly-4vinylpyridine (PS-b-P4VP) type. The outer surface of HNTs was modified by physical adsorption of SDS and BCP molecules, which gave a stable suspension of HNTs in PS solutions and a good dispersion in the final PS nanocomposite films after evaporation of the solvent. XRD was used to study the crystal structure of pristine HNTs. Fourier-transform infrared spectroscopy (FT-IR) and TGA prove the successful modification of HNTs with SDS and BCP molecules. The microstructure of pristine and modified HNTs was investigated with field-emission scanning electron microscopy (FESEM) and high resolution transmission electron microscopy (HR-TEM). TGA and DSC were used to study the structural decomposition and the crystallisation temperature of PS/HNT nanocomposites. Thin sections of PS/HNT nanocomposites were prepared at room temperature via ultramicrotomy and TEM micrographs were taken showing the effect of the noncovalent modification on the dispersion of HNTs in the PS matrix. The variation of absorbance and transmittance of the PS/HNT nanocomposite films with respect to neat PS film in the UV and visible range of light was studied by UV-vis spectroscopy. The excellent UV absorbance blocking properties of the nanocomposite films suggest their potential use as UV-filters.

\section{Experimental}

2.1. Materials. Polystyrene (PS) with average molecular weight $\left(M_{w}\right)$ of $M_{w} \sim 400,000$ was purchased from Sigma Aldrich and used as the matrix for the fabrication of PS/HNT nanocomposite films. Halloysite nanotubes (aspect ratio: $\mathrm{L} / \mathrm{D}$ $=4$, according to the supplier's data) and sodium dodecyl sulphate (SDS) were obtained from OmniPur. PS-b-P4VP (product ID: P4829P-S4VP) diblock copolymer with the following molecular characteristics, $M_{w} / M_{n}=1.05, M_{n}\left(\times 10^{3}\right)$ $=50.0-\mathrm{b}-13.0$, was obtained from Polymer Source. Tetrahydrofuran (THF), the solvent for preparing the PS solutions and the respective PS/HNT nanocomposites, was obtained from Sigma Aldrich. All chemicals were of analytical grade and used as received without further purification.

2.2. Modification of HNTs with SDS and BCP and Fabrication of PS/HNT Nanocomposite Films. In order to modify HNTs with the anionic surfactant SDS, $0.02 \mathrm{wt} \%$ of HNTs were mixed with 1 wt. $\%$ of SDS aqueous solution, stirred for $24 \mathrm{~h}$, and sonicated for $5 \mathrm{~h}$. The mixture was kept to stand for $24 \mathrm{~h}$ and the supernatant HNTs were collected, centrifuged, and dried in a vacuum oven at $70^{\circ} \mathrm{C}$ for $24 \mathrm{~h}$. For modification of HNTs by BCP, a solution of PS-b-P4VP with (1 wt.\%) was prepared in THF and the same amount of HNTs was added and sonicated for $3 \mathrm{~h}$. The solutions were kept to stand for $24 \mathrm{~h}$, and the supernatant HNTs were collected, centrifuged, and dried in a vacuum oven at $50^{\circ} \mathrm{C}$ for $24 \mathrm{~h}$.

For the PS nanocomposites, initially homopolymer PS was dissolved in THF, $10 \%(\mathrm{w} / \mathrm{v})$. Then, 1,2 , and $5 \mathrm{wt} . \%$ of SDS-m-HNT and BCP-m-HNT were added to the PS solutions in THF (wt.\% with respect to the polymer mass in the solution). These solutions were sonicated for $10 \mathrm{~min}$ and then magnetically stirred for $1 \mathrm{~h}$. Finally, the PS/HNT solutions 


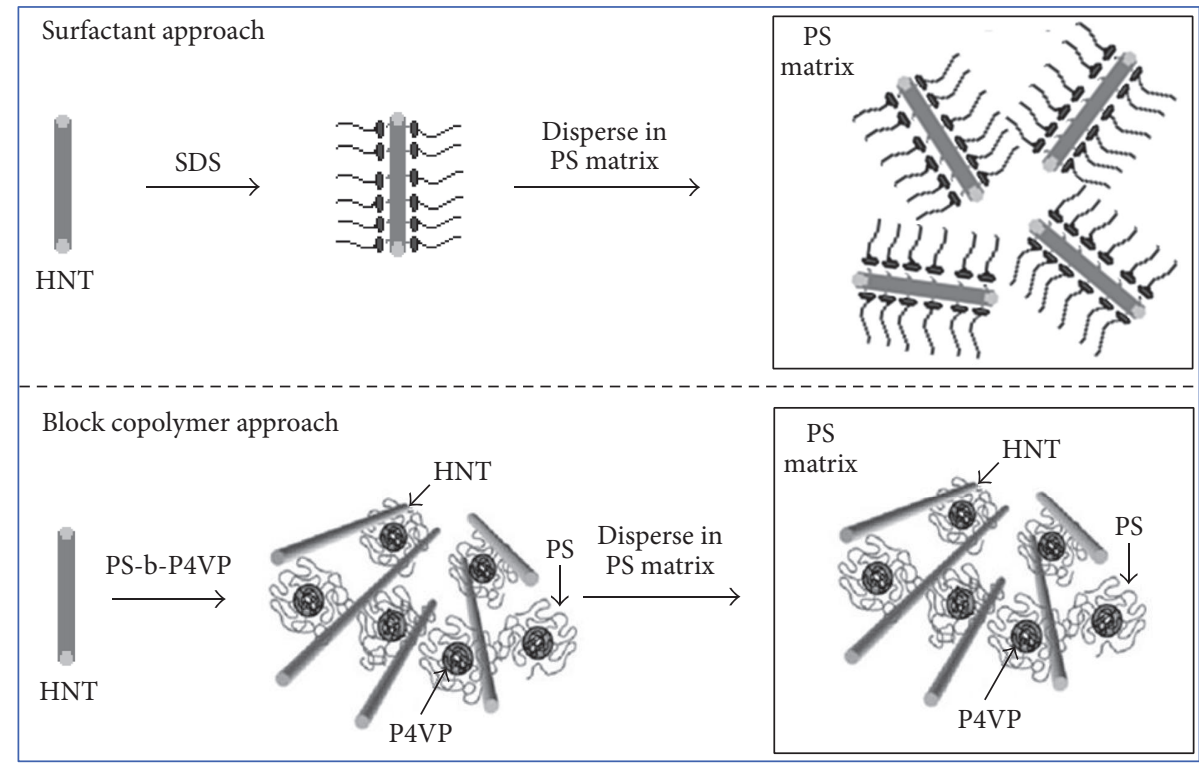

FIGURE 1: Schematic diagram showing the mechanism for the physical adsorption of SDS and BCP molecules onto the surface of HNTs and their effective dispersion in PS matrix.

were casted in Teflon moulds and dried at room temperature for $48 \mathrm{~h}$. Finally, all the nanocomposite films were inserted in a vacuum oven and kept at $50^{\circ} \mathrm{C}$ under vacuum overnight to fully remove traces of solvent and induce a thermal annealing effect to the nanocomposite films for the better thermodynamic organisation of the polymer chains.

Figure 1 demonstrates the mechanism for the physical adsorption (noncovalent functionalisation) of SDS and BCP molecules to the surface of HNTs and the affinity of the organic pending groups towards the effective dispersion of HNTs in the PS matrix.

2.3. Characterisation Techniques. XRD was performed with an X-ray diffractometer XRD T/T (GE Inspection Technologies Ahrensburg, Germany) in symmetric step-scan mode with $\Delta 2 \Theta=0.05^{\circ}$ in transmission operating at $40 \mathrm{kV}$ and $30 \mathrm{~mA}$ with $\mathrm{Cu} \mathrm{K} \alpha$ radiation. FT-IR spectra were recorded using a Vertex 80v FT-IR spectrometer (Bruker Germany) equipped with a DTGS detector by signal averaging of 256 scans. Approximately $1.0 \mathrm{mg}$ of pristine HNTs, SDS-mHNT, and BCP-m-HNT was pressed together with $100 \mathrm{mg}$ of crystalline $\mathrm{KBr}$ to form pellets. The pellets were scanned over the range $4000-400 \mathrm{~cm}^{-1}$ at a resolution of $\pm 2 \mathrm{~cm}^{-1}$. Thermal analysis experiments were carried out using DSC and TGA. TGA studies were performed using a TA instrument (Q 5000) for the HNTs and PS/HNT nanocomposites. The temperature range varied from ambient $\left(25^{\circ} \mathrm{C}\right.$ ) up to $800^{\circ} \mathrm{C}$ (for samples of approx. $10 \mathrm{mg}$ ), with $2 \mathrm{~K} / \mathrm{min}$ heating rate, under nitrogen $\left(\mathrm{N}_{2}\right)$ atmosphere. Curie point standards were utilized for the temperature calibration. The initial degradation temperatures and the temperatures corresponding to 50\% degradation of the samples were recorded. DSC measurements were carried out using $5-10 \mathrm{mg}$ of PS/HNT nanocomposite samples to determine the glass transition temperatures $\left(T_{g}\right)$ of the films. For the analysis, a DSC Q 1000 from TA Instruments was used at a $10 \mathrm{~K} / \mathrm{min}$ heating rate under nitrogen atmosphere. The samples were heated to $140^{\circ} \mathrm{C}$, held for 5 minutes for removing moisture and previous thermal history, and then cooled to room temperature at a cooling rate of $10^{\circ} \mathrm{C} / \mathrm{min}$. The second heating scans were used for determination of glass transition temperature of the samples. FE-SEM was performed using the NEON 40 (Carl Zeiss AG, Germany) scanning electron microscope. All the images were generated using the secondary electron detector (SE2) under an accelerating voltage of $1.0 \mathrm{kV}$. Samples were prepared by drop-casting $100 \mu \mathrm{L}$ of pristine and modified HNTs on a $2 \times 1 \mathrm{~cm}^{2}$ silicon substrate followed by drying at room temperature in a fume hood. TEM investigations were performed to study the geometry of HNTs and the microstructures of PS/HNT nanocomposite films using the Libra 200 transmission electron microscope (Carl Zeiss AG, Germany) operating at $200 \mathrm{kV}$. Samples for TEM were prepared by dispensing $10 \mu \mathrm{L}$ of pristine HNTs on a $\mathrm{Cu}$ grid with a carbon support membrane followed by drying. Thin sections of approximately $70 \mathrm{~nm}$ thickness were obtained by ultramicrotomy using a Leica Ultramicrotome. Reichert Ultracut S. Diamond knives (Diatome) were used for the trimming (model cryotrim $45^{\circ}$ ) as well as the thin-section cutting process (model cryo $35^{\circ}$ ) and all preparation steps were done at room temperature as described elsewhere [3941]. The thin sections were placed on copper grids (300 mesh $\mathrm{Cu}$, Agar) and sputtered with a $10 \mathrm{~nm}$ thickness carbon layer to avoid charging effects and protect the samples from the electron beam [42]. The variation of absorbance and transmittance of the PS/HNT nanocomposite films containing 1, 2, and 5 wt.\% SDS and BCP modified HNTs with respect to pure PS film in the UV and visible range of light was studied at room temperature using a Perkin-Elmer Lambda $800 \mathrm{UV}$-vis spectrophotometer (with an incorporated xenon flash lamp). The variation of transmittance of nanocomposites films with the amount of HNTs is also studied and discussed in detail. 


\section{Results and Discussion}

\subsection{Characterisation of HNTs}

3.1.1. X-Ray Diffraction Analysis of Pristine and Modified HNTs. Figure 2 shows the XRD pattern of pristine HNTs, as well as SDS-m-HNT and BCP-m-HNT. Indexing can be done based on the presence of two different crystalline phases, namely, the halloysite and the kaolinite $\left[\mathrm{KAl}_{3}\left(\mathrm{SO}_{4}\right)_{2}(\mathrm{OH})_{6}\right]$ crystal structure. The close structural relationship between them gives rise to similar powder diffraction patterns. Over the years, this subject has been a big issue in the literature and nowadays it is generally accepted that, in the case of halloysite, the intensity ratio between the 002 and 020/110 reflections should be $\sim \leq 2$, which is true in case of our pristine HNT sample. The pure HNTs show a diffraction peak at $2 \theta=12.25$, which is related to the $\left(\begin{array}{lll}0 & 0 & 1\end{array}\right)$ plane. This basal reflection of HNTs is coming from its tubular morphology, high degree of disorder, small crystal size, and interstratifications of layer with various hydration states. This peak of pristine HNTs is shifted to a lower $2 \theta$ value for the SDS-m-HNT and BCP-mHNT, respectively. The $d$-spacing of SDS-m-HNT is $0.73 \mathrm{~nm}$ at a $2 \theta$ of 12.15 . The value of $2 \theta$ is even lower for the BCP-mHNT sample, which is 11.85 corresponding to the $0.745 \mathrm{~nm}$ basal spacing. The observed reduction of $2 \theta$ corresponding to increased basal spacing of HNTs may be attributed to the intercalation of HNTs by SDS surfactant molecules and BCP micelles physical adsorption, respectively. Apart from the aforementioned peak at low $2 \theta$, the diffraction pattern of pristine HNT is showing other peaks at $2 \theta$ of 20.06 and 24.94, respectively, which are related to $\left(\begin{array}{lll}0 & 2 & 0\end{array}\right)$ and $\left(\begin{array}{lll}0 & 0 & 2\end{array}\right)$ basal reflection. It should be noted that the peak at 20.06 almost vanishes in the SDS-m-HNT and BCP-m-HNT, while the peak at 24.94 is shifted to lower $2 \theta$ value supporting the intercalation of SDS molecules and BCP chains into the HNT gallery.

3.1.2. FT-IR Spectra of Pristine and Modified HNTs. FT-IR spectra of pristine, SDS, and BCP modified HNTs are shown in Figure 3. In FT-IR spectra of pristine HNTs, there is a very strong absorption at $1000 \mathrm{~cm}^{-1}$ due to the $\mathrm{O}-\mathrm{Si}-\mathrm{O}$ siloxane surface groups confirming of HNTs. Also the Al-OH peaks at 3694,3626 , and $3623 \mathrm{~cm}^{-1}$ and $\mathrm{Si}-\mathrm{O}$ vibrations at 1119 and $1000 \mathrm{~cm}^{-1}$ were observed, which are characteristic peaks of HNTs. The following peaks are also seen: $906 \mathrm{~cm}^{-1}$ corresponding to $\mathrm{O}-\mathrm{H}$ deformation of inner hydroxyl groups, $796 \mathrm{~cm}^{-1}$ corresponding to symmetric stretching of $\mathrm{Si}-\mathrm{O}$, and $684 \mathrm{~cm}^{-1}$ corresponding to perpendicular Si-O stretching. In the case of SDS-m-HNT, a peak corresponding to $\mathrm{SO}_{3}{ }^{-}$ stretch at 1217 and $1248 \mathrm{~cm}^{-1}, \mathrm{CH}_{2}$ asymmetric stretch at 2917, $\mathrm{CH}_{2}$ symmetric stretch at 2850 , and $\mathrm{CH}_{2}$ rocking mode at $721 \mathrm{~cm}^{-1}$ could be observed apart from the characteristic peaks of HNTs. The additional characteristic peaks of pyridine ring of PS-P4VP at 1416 and $1598 \mathrm{~cm}^{-1}$ are present in the BCP modified HNT sample. Figure 3(b) shows the colloidal stability of SDS and BCP modified HNTs well dispersed in the PS solution where no precipitation has been observed within $24 \mathrm{~h}$. In case of SDS modification, the anionic surfactant

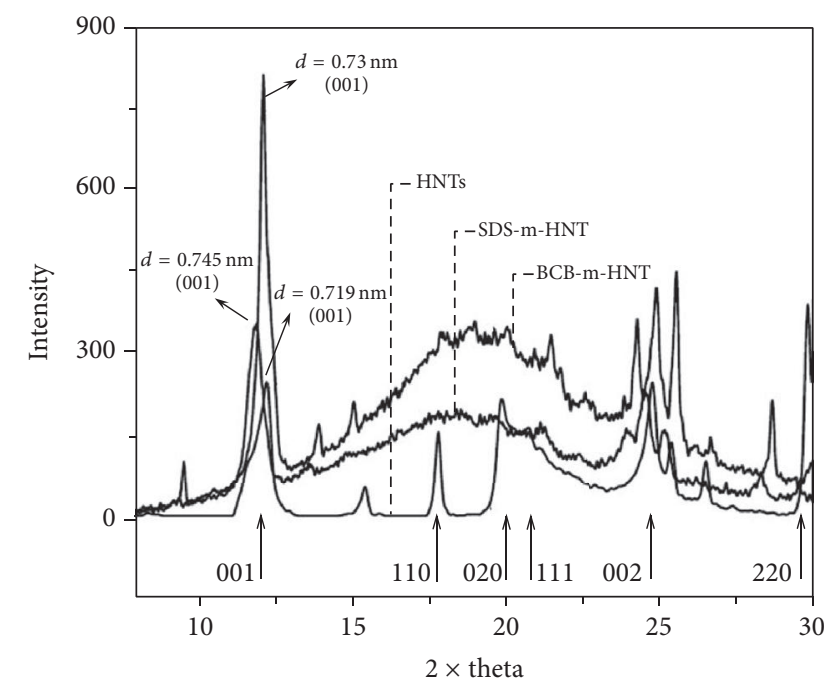

FIGURE 2: XRD pattern of pristine and modified HNTs.

forms a bilayer on the surface of HNTs making it organophilic and dispersible in the polymer matrix. The PS-b-P4VP as an amphiphilic block copolymer forms micelles, which act as dispersants by physically adhering to the surface of the HNTs.

3.1.3. TGA Analysis of Pristine, BCP, and SDS Modified HNTs. Figure 4(a) shows the TGA and Figure 4(b) the derivative of weight loss for pristine, BCP, and SDS modified HNTs demonstrating their structural decomposition process and as a means to prove the noncovalent attachment of SDS and BCP molecules. As can be seen, the decomposition of pristine HNTs takes place in two main steps. At first, the endothermic peak around $100^{\circ} \mathrm{C}$ is due to the moisture present in the sample (1.6 wt.\%). Structural decomposition of halloysite and alunite takes place in the second endothermic peak from 400 to $550^{\circ} \mathrm{C}(15.8 \mathrm{wt} . \%)$, and a residue of $82.8 \mathrm{wt} . \%$ was found at $650^{\circ} \mathrm{C}$. In Figure $4(\mathrm{~b})$, also the decomposition peaks of pristine HNTs (near to $100^{\circ} \mathrm{C}$ ) corresponding to evaporation of moisture and the other at $450^{\circ} \mathrm{C}$ corresponding to structural decomposition of HNTs are observed. SDS modified HNTs show first weight loss at $200^{\circ} \mathrm{C}$ corresponding to SDS (27.5 wt.\%), while decomposition of halloysite and alunite takes place in the second endothermic peak from 400 to $550^{\circ} \mathrm{C}\left(16.2\right.$ wt.\%) with a final residue of 44.5 wt. $\%$ at $650^{\circ} \mathrm{C}$. From Figure 4(b) DTG graph, for SDS modified HNTs first peak near $200^{\circ} \mathrm{C}$ corresponding to decomposition of SDS and the second at $420^{\circ} \mathrm{C}$ corresponding to decomposition of HNT can be seen. BCP modified HNTs show weight loss between 200 and $450^{\circ} \mathrm{C}$ with a final residue of $44.6 \mathrm{wt} . \%$ at $650^{\circ} \mathrm{C}$. Considering the decomposition of halloysite and alunite from 400 to $550^{\circ} \mathrm{C}(16.0-16.5 \mathrm{wt} . \%)$, it is estimated that the amount of BCP chains noncovalently bound onto the surface of HNTs is approximately 37 wt.\%. From Figure 4(b) for BCP modified HNTs, there is one large peak at around $400^{\circ} \mathrm{C}$ due to overlap of decomposition temperatures of PSb-P4VP BCP and HNTs. 


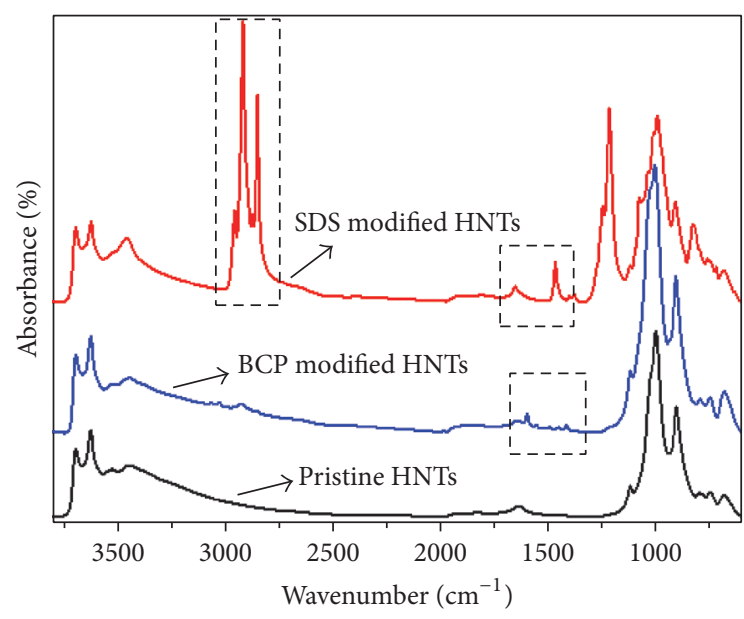

(a)

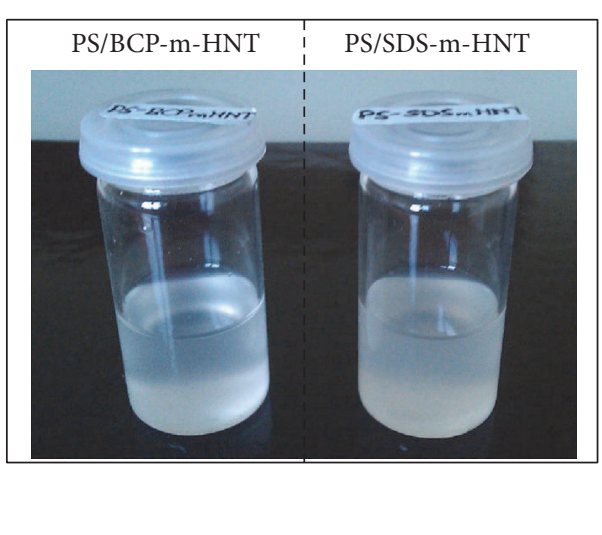

(b)

FIGURE 3: (a) Infrared spectra of pristine, BCP, and SDS modified HNTs; (b) PS solution containing BCP and SDS modified HNTs.

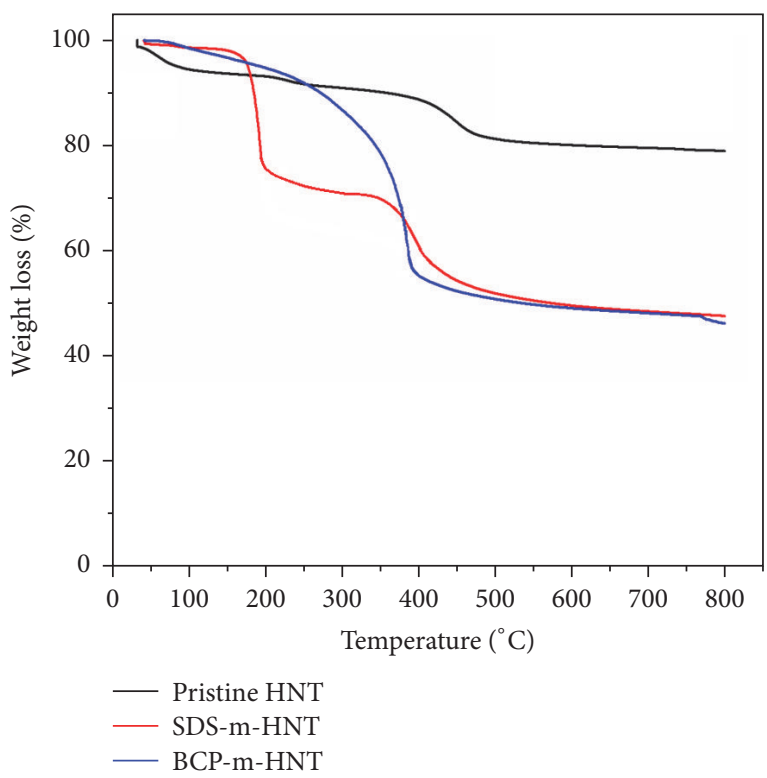

(a)

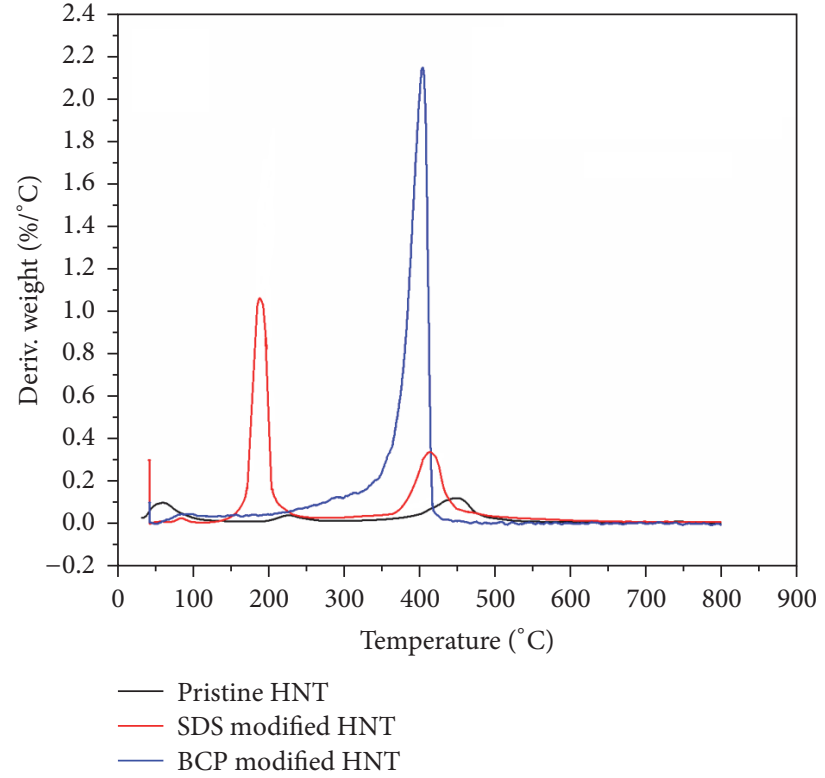

(b)

FIgURE 4: (a) TGA and (b) derivative of weight loss (DTG) of pristine, SDS, and BCP modified HNTs.

3.1.4. Scanning and Transmission Electron Microscopy of HNTs. Figure 5 shows the SEM images of pristine (Figures 5(a) and 5(b)), SDS modified (Figure 5(c)), and BCP modified (Figure 5(d)) HNTs, respectively. The diameters of pristine HNTs are found out to be in the range of 70-100 nm and the lengths in the range of 180-1300 nm. In Figure 5(d), it is observed that HNTs are wrapped by the BCPs molecules. Figures 6(a) and 6(b) are TEM images at two different magnifications depicting the geometrical characteristics of HNTs (HNT length and diameter), which as can be seen are in good agreement with the SEM investigations.

3.2. Characterisation of PS-HNT Nanocomposite Films. PSHNT nanocomposite films were subjected to TGA analysis (under $\mathrm{N}_{2}$ atmosphere) in order to analyse the effect of HNT loading on the thermal stability and potential improvement. The results of TGA and DTG of PS-HNT nanocomposites containing SDS modified HNTs are shown in Figures 7(a) and 7(b), respectively. It is observed that the temperature required for $10 \%$ degradation, termed as the degradation onset temperature $\left(T_{0.1}\right)$, is found to be increased from $250^{\circ} \mathrm{C}$ for pure PS film to $262^{\circ} \mathrm{C}, 276^{\circ} \mathrm{C}$, and $289^{\circ} \mathrm{C}$ for nanocomposite films containing 1,2 , and $5 \%$ SDS modified HNTs, respectively (values given in Table 1). Also, the temperature required for $50 \%$ degradation $\left(T_{0.5}\right)$ is increased from $385^{\circ} \mathrm{C}$ for pure PS film to $394^{\circ} \mathrm{C}, 400^{\circ} \mathrm{C}$, and $410^{\circ} \mathrm{C}$ for nanocomposite films containing 1,2 , and $5 \%$ SDS modified HNTs. It can be concluded that the thermal stability of PS nanocomposite 


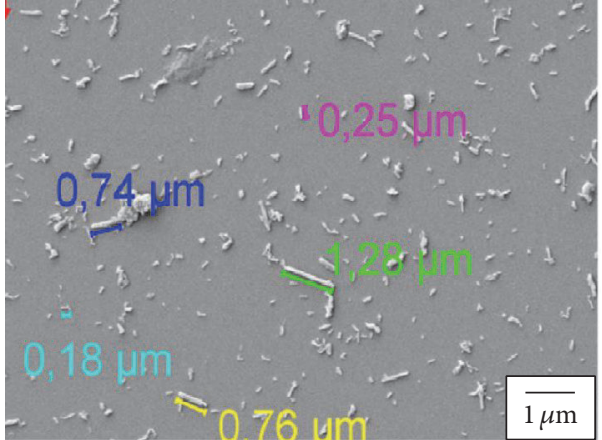

(a)

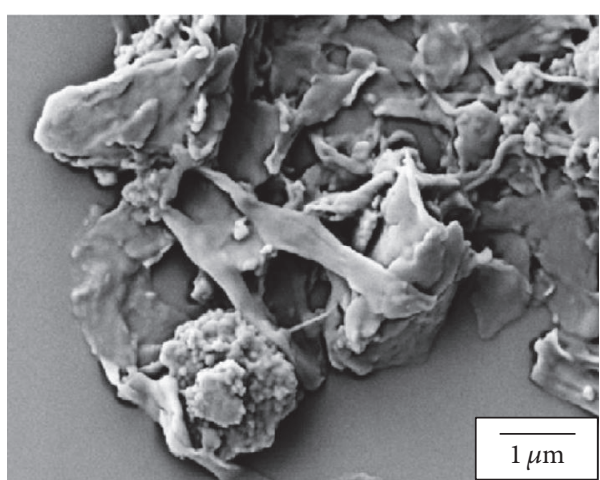

(c)

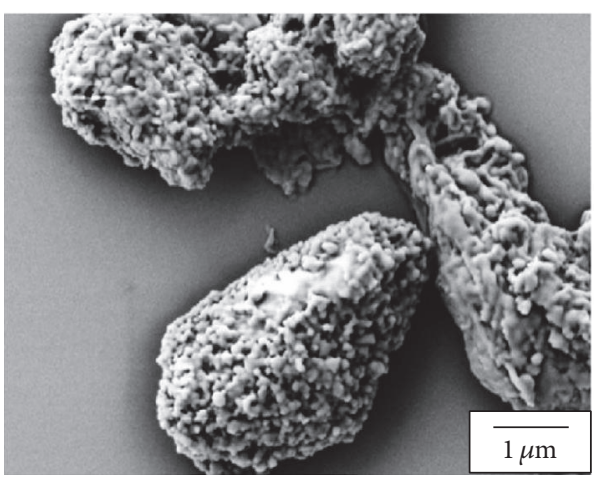

(e)

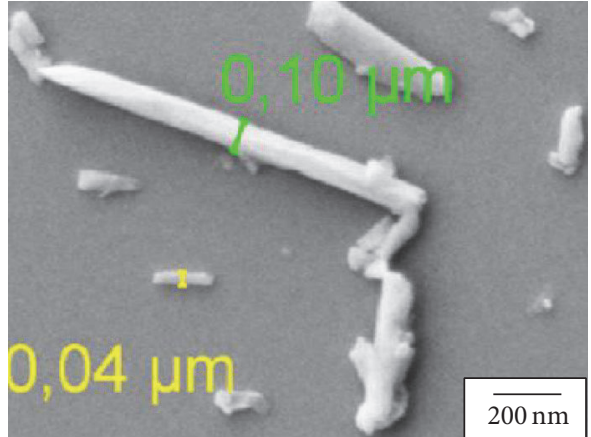

(b)

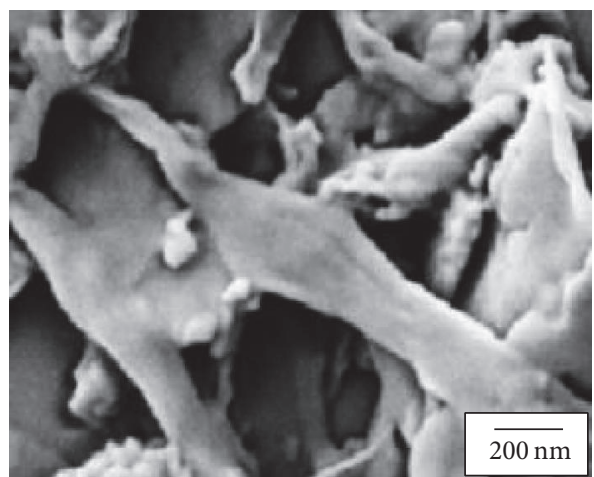

(d)

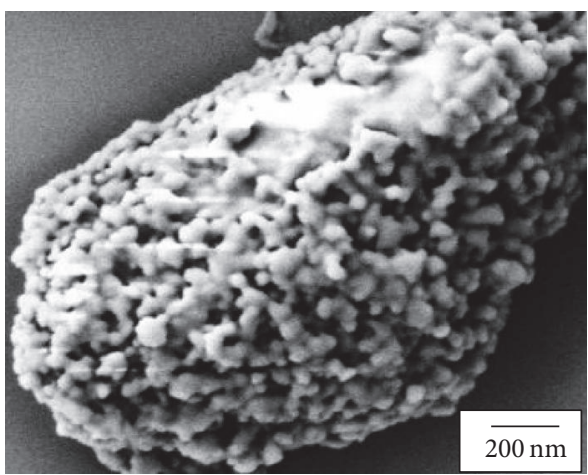

(f)

FIGURE 5: SEM micrographs of pristine HNTs (a, b); (c, d) SDS modified HNTs; and (e, f) BCP modified HNTs at two different magnifications.

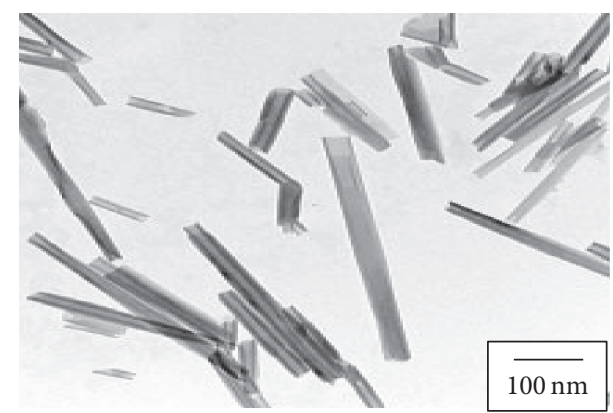

(a)

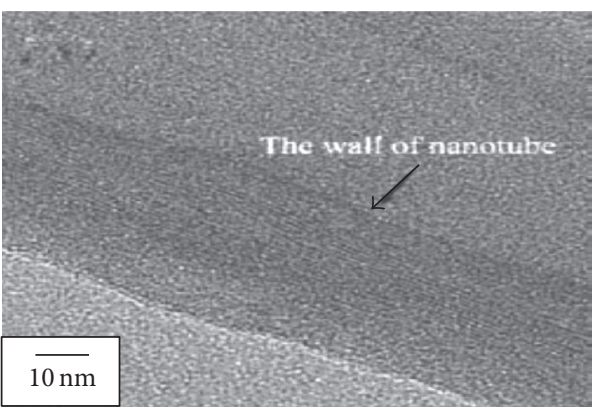

(b)

FIGURE 6: TEM micrographs of pristine HNTs at two different magnifications. 


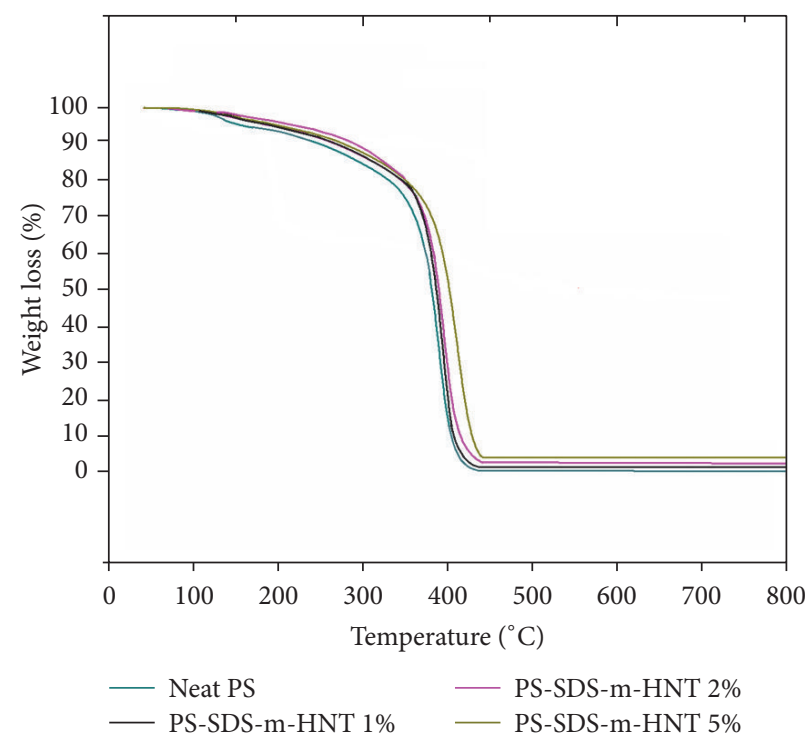

(a)

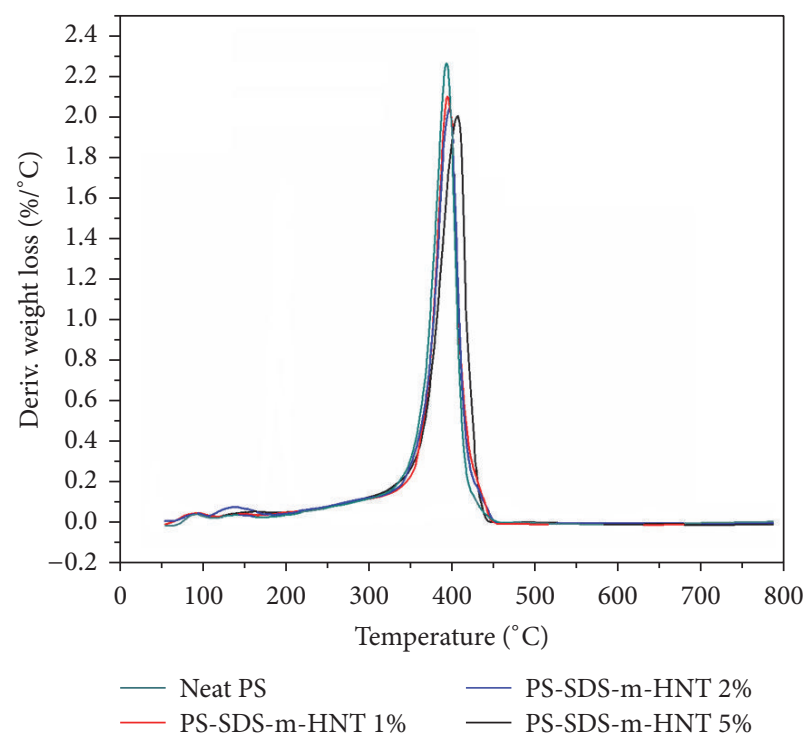

(b)

Figure 7: (a) TGA and (b) DTG of PS-HNT nanocomposites with SDS modified HNTs.

TABLE 1: Temperatures corresponding to onset of degradation $\left(T_{0.1}\right)$ and temperatures at $50 \%$ degradation $\left(T_{0.5}\right)$ with respect to wt.\% of SDS modified HNTs in the PS-HNT nanocomposite films.

\begin{tabular}{lcc}
\hline wt.\% SDS modified HNT & $T_{0.1}\left({ }^{\circ} \mathrm{C}\right)$ & $T_{0.5}\left({ }^{\circ} \mathrm{C}\right)$ \\
\hline & 250 & 385 \\
1 & 262 & 394 \\
2 & 276 & 400 \\
5 & 289 & 410 \\
\hline
\end{tabular}

TABLE 2: Temperatures corresponding to onset of degradation $\left(T_{0.1}\right)$ and temperatures at $50 \%$ degradation $\left(T_{0.5}\right)$ with respect to wt.\% of BCP modified HNTs in the PS-HNT nanocomposite films.

\begin{tabular}{lcc}
\hline wt.\% BCP modified HNT & $T_{0.1}\left({ }^{\circ} \mathrm{C}\right)$ & $T_{0.5}\left({ }^{\circ} \mathrm{C}\right)$ \\
\hline & 250 & 385 \\
1 & 257 & 390 \\
2 & 265 & 395 \\
5 & 282 & 400 \\
\hline
\end{tabular}

films is higher than that of pure PS film and it increases with the increase of the SDS modified HNT loadings in the nanocomposite films. The entrapment of decomposition products by the HNT lumen may be responsible for the improved thermal degradation of PS nanocomposite films.

The results of TGA and DTG analysis of PS nanocomposites containing BCP modified HNTs are shown in Figures 8(a) and $8(\mathrm{~b})$, respectively. It is can be clearly seen that the thermal degradation (corresponding to $T_{0.1}$ ) in the case of pure PS film starts at $250^{\circ} \mathrm{C}$, whereas for the nanocomposite films containing 1,2 , and $5 \% \mathrm{BCP}$ modified $\mathrm{HNTs}$ at $257^{\circ} \mathrm{C}, 265^{\circ} \mathrm{C}$, and $282^{\circ} \mathrm{C}$, respectively (Table 2 ). Also the temperature corresponding to $50 \%$ degradation $\left(T_{0.5}\right)$ in the case of PS film is found to be $385^{\circ} \mathrm{C}$, whereas for nanocomposite films containing 1,2 , and $5 \% \mathrm{BCP}$ modified $\mathrm{HNTs}$ at $390^{\circ} \mathrm{C}, 395^{\circ} \mathrm{C}$, and $400^{\circ} \mathrm{C}$, respectively. Hence, it can be concluded that the nanocomposite films show improved thermal stability compared to pure PS film for potential reasons explained above.

Figure 9 shows the TEM micrographs at two different magnifications (10 K and $16 \mathrm{~K}$ mag.) for the PS-HNT nanocomposites filled with $1 \mathrm{wt} . \%$ of pristine (Figures 9(a) and 9(d)) as well as SDS (Figures 9(b) and 9(e)) and BCP (Figures $9(\mathrm{c})$ and $9(\mathrm{f})$ ) modified HNTs. The images reveal that the nanocomposite films containing modified HNTs exhibit better dispersion in the PS matrix compared to the pristine HNTs. However, local aggregation of HNTs is also seen at some points, and this aggregation is more pronounced in the case of BCP modified HNTs. Therefore, it can be concluded that SDS modified HNTs give better global distribution of HNTs in the polymer matrix.

Figure 10(a) shows the UV-vis spectra of PS-HNT nanocomposite films with different HNT loadings as a means to study their optical transparency. It can be observed that the onset of absorbance for pure PS film, as well as for the nanocomposite films, is in the range of $200-300 \mathrm{~nm}$. The absorbance values for all the films are very low in the visible region, which indicates that the prepared nanocomposite films do not possess any color and can readily be used as transparent UV-absorbing materials. The absorbance of all the nanocomposite films is slightly higher than that of pure PS film. Also, as the amount of BCP and SDS modified HNTs increases from 1 to $5 \mathrm{wt}$.\%; the absorbance increases in the $\mathrm{UV}$ as well as in the visible range. It should be also mentioned that the absorbance values of nanocomposite films containing 1,2 , and 5\% BCP modified HNTs are higher than that of nanocomposite films containing 1,2, and 5\% SDS modified HNTs. This is due to presence of $\pi$-electrons present in the BCP (PS-b-P4VP). More specifically, molecules containing $\pi$-electrons or nonbonding electrons (n-electrons) can 


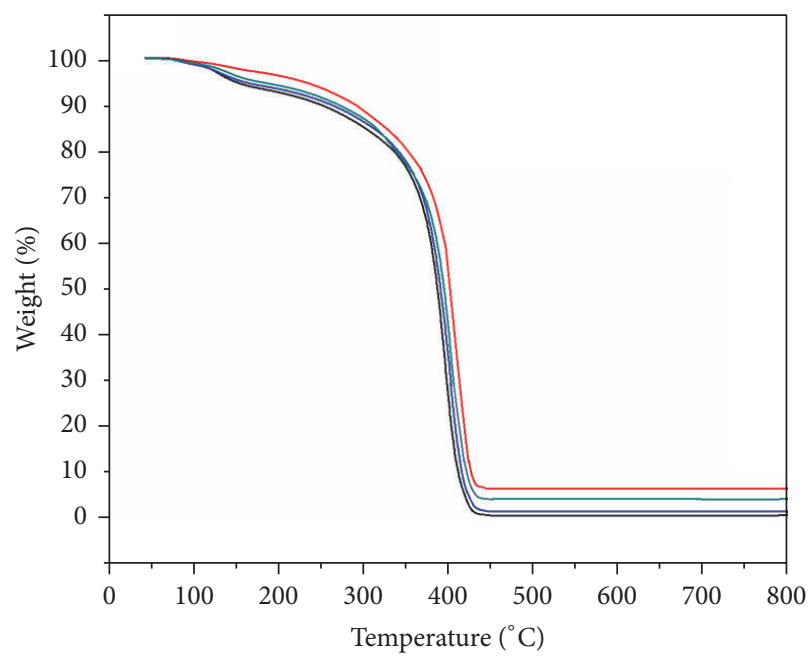

$\begin{array}{ll}\text { — Pure PS film } \% & \text { PS-BCP-m-HNT } 2 \% \text { film } \\ \text { PS-BCP-m-HNT 1\% film } & \text { PS-BCP-m-HNT } 5 \% \text { film }\end{array}$

(a)

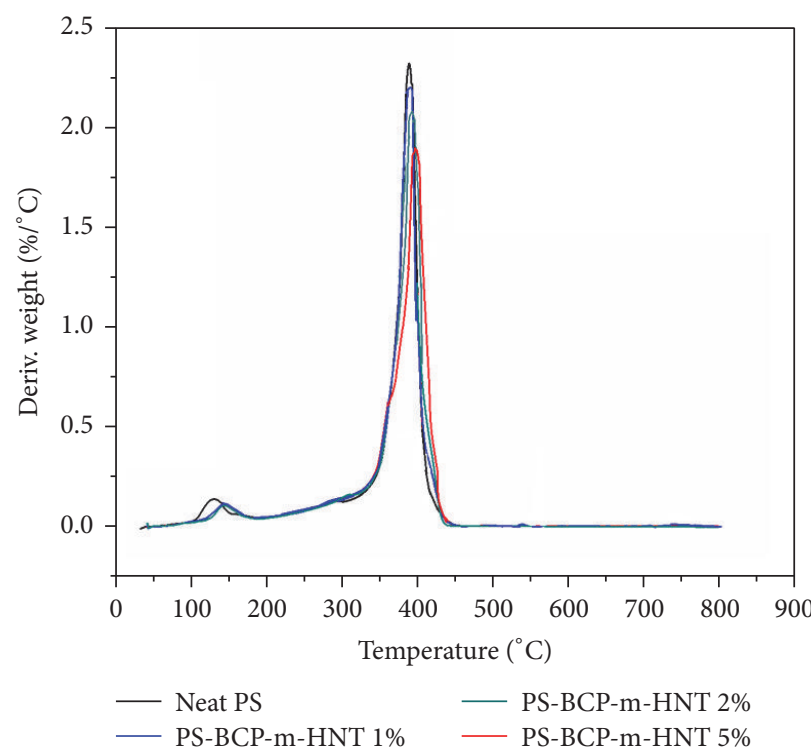

(b)

FIGURE 8: (a) TGA and (b) DTG of PS-HNT nanocomposites with BCP modified HNTs.

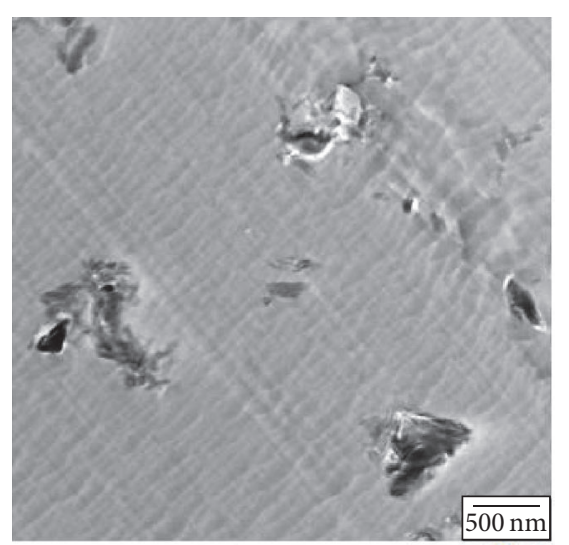

(a)

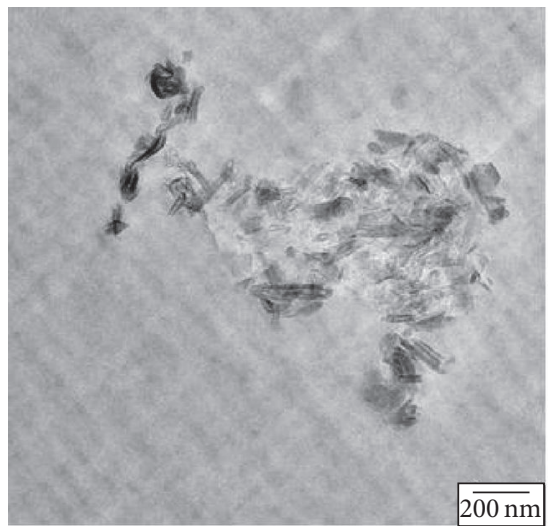

(d)

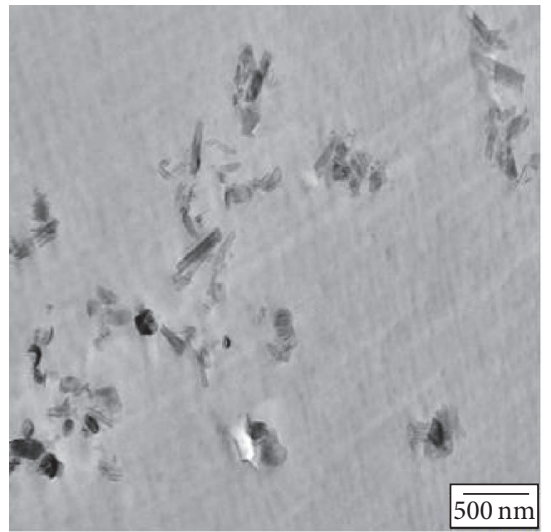

(b)

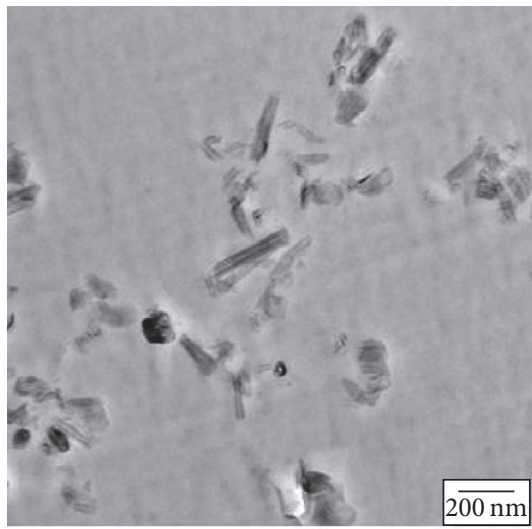

(e)

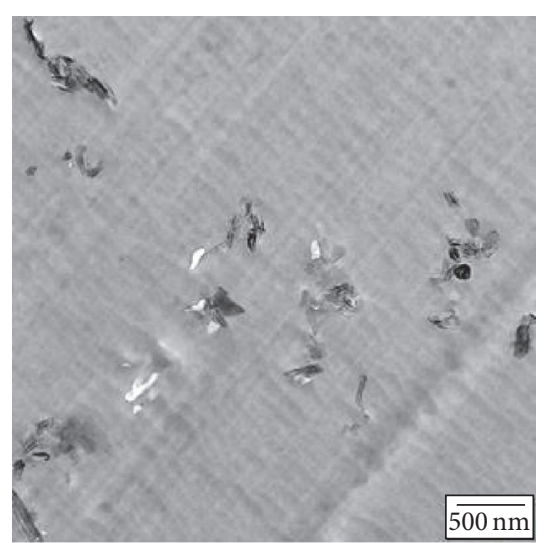

(c)

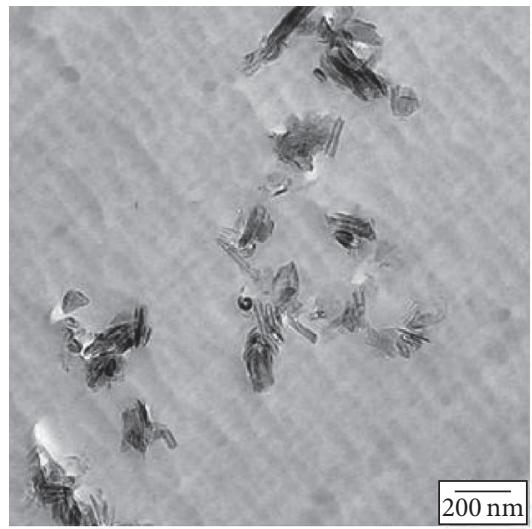

(f)

FIGURE 9: TEM micrographs at two different magnifications of PS-HNT nanocomposites containing (a) pristine, (b) SDS, and (c) BCP modified HNTs, respectively, at $1 \mathrm{wt} . \%$ loadings. 

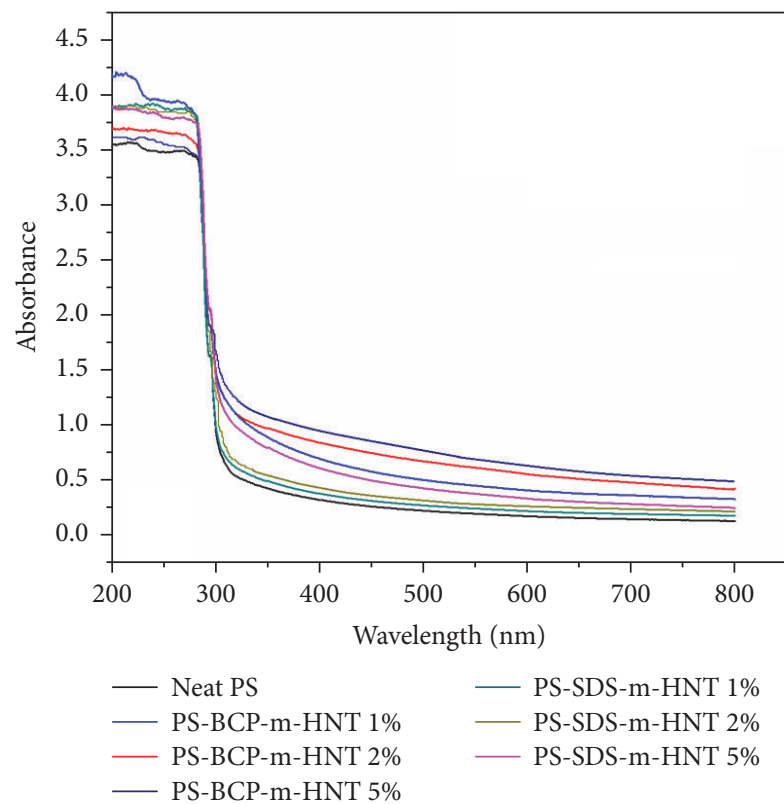

(a)
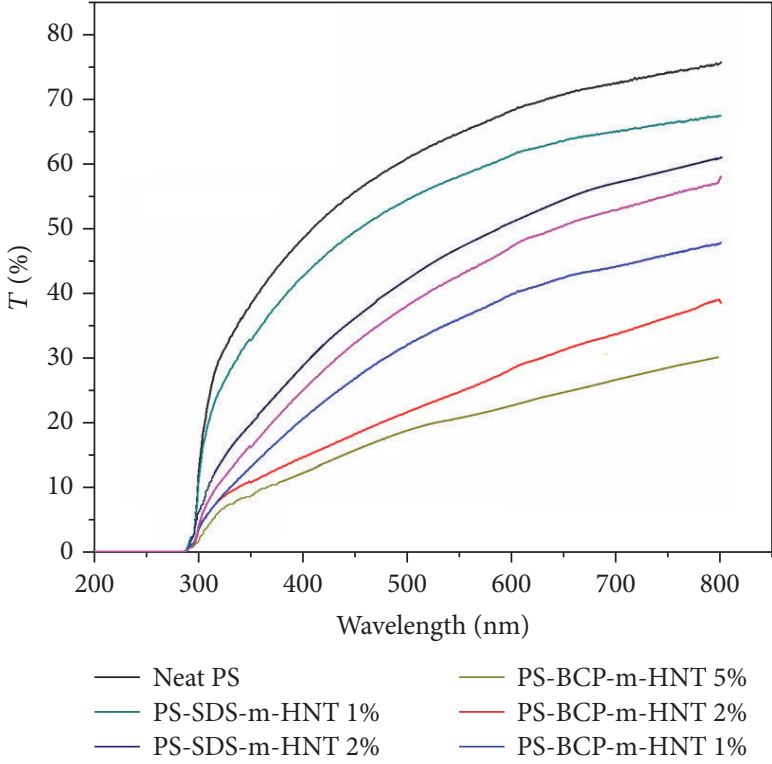

(b)

FIGURE 10: (a) UV-vis spectra of PS-HNT nanocomposite films with BCP modified HNTs and SDS modified HNTs compared to pure PS film; (b) \% transmittance of PS-HNT nanocomposite films with BCP modified HNTs and SDS modified HNTs compared to neat PS film.

TABLE 3: Variation of $\% T$ in case of pure PS film and the nanocomposite films containing SDS modified HNTs with the amount of HNTs (no variations in the optical properties for SDS/PS films have been found compared to neat PS).

\begin{tabular}{lcc}
\hline$\%$ HNT & $\begin{array}{c}\text { \% } \text { in UV region } \\
(350 \mathrm{~nm})\end{array}$ & $\begin{array}{c}\% T \text { in visible } \\
\text { region }(550 \mathrm{~nm})\end{array}$ \\
\hline 0 & 38.28 & 64.72 \\
1 & 32.66 & 58.63 \\
2 & 19.56 & 45.10 \\
5 & 16.17 & 42.73 \\
\hline
\end{tabular}

absorb the energy in the form of ultraviolent or visible light to excite these electrons to higher antibonding molecular orbitals.

Figure 10 (b) shows the variation of $\% T$ (optical transmittance) of the nanocomposite films as a function of wavelength. It is observed that the transmission starts from $300 \mathrm{~nm}$ onwards and it decreases for all nanocomposites films compared to pure PS film. The variation of $\% T$ with the amount of HNTs in the nanocomposite films is also studied.

Tables 3 and 4 give the exact data of variation of $\% T$ with wt.\% loading of modified HNT in the nanocomposite films at two different wavelengths: $350 \mathrm{~nm}$ (corresponding to $\mathrm{UV}$ region) and $550 \mathrm{~nm}$ (corresponding to visible range). It is observed that the \% transmittance decreases as \% BCP modified HNTs and SDS modified HNTs in the nanocomposites films increase.

By comparing the values in Tables 3 and 4 , it can be concluded that the decrease in $\% T$ of nanocomposite films with respect to pure PS films is more in the case of films containing
TABLE 4: Variation of $\% T$ in case of pure PS film and the nanocomposite films containing BCP modified HNTs with the amount of HNTs (no variations in the optical properties for BCP/PS films have been found compared to neat PS).

\begin{tabular}{lcc}
\hline$\%$ HNT & $\begin{array}{c}\text { \% in UV region } \\
(350 \mathrm{~nm})\end{array}$ & $\begin{array}{c}\% T \text { in visible } \\
\text { region }(550 \mathrm{~nm})\end{array}$ \\
\hline 0 & 38.27 & 64.71 \\
1 & 13.18 & 36.05 \\
2 & 10.82 & 24.78 \\
5 & 8.40 & 20.01 \\
\hline
\end{tabular}

BCP modified HNTs than that of films containing SDS modified HNTs. Therefore, in order to retain the transparency of nanocomposites films, the SDS modification of HNTs proves to be better than the BCP modified HNTs.

\section{Conclusions}

In this study, HNTs were successfully modified by SDS and BCP (PS-b-P4VP) organic materials via noncovalent bonds. This facilitated the reduction of inherent hydrophilicity of HNTs making them compatible with the PS polymer matrix. The modifications of HNTs are found to give good dispersion of nanofiller, that is, HNT into the host polymer matrix as shown by the TEM analysis. Hence, the incorporation of modified HNTs in PS matrix for the fabrication of PS nanocomposite films was very successful. A reasonable increase in thermal and optical properties of PS films was observed after loading with small amounts of modified HNTs. The effect of increasing the amount of HNTs in nanocomposite 
films on these properties was also studied. It is found that as the loading of HNTs was increased in the PS films, the thermal stability increased without losing much of optical transparency. Since the nanocomposite films are highly transparent and possess good absorption of light in the UV region, these nanocomposites films can be used as transparent and UV shielding materials (greenhouses, food packaging, optical lenses, etc.). Also, comparing the dispersion of HNTs in host matrix and the thermal and optical properties of the nanocomposite films, we conclude that the nanocomposite films containing SDS modified HNTs show better results in all aspects compared to films containing BCP modified HNTs. The modification methods proposed in this study may help in exploiting the potential of HNTs in other polymer matrices due to the achieved HNT dispersion.

\section{Conflicts of Interest}

The authors declare that there are no conflicts of interest regarding the publication of this paper.

\section{Acknowledgments}

The authors would like to thank Dr. M. Malanin and Mrs. L. Hässler for FT-IR and DSC experimental support, respectively. Also grateful thanks are due to Dr. Amit Das for the supply of the halloysite nanotubes. Lazaros Tzounis gratefully acknowledges the Bodossaki Foundation for financial support.

\section{References}

[1] H. Fischer, "Polymer nanocomposites: from fundamental research to specific applications," Materials Science and Engineering C, vol. 23, no. 6-8, pp. 763-772, 2003.

[2] J. K. Pandey, K. Raghunatha Reddy, A. Pratheep Kumar, and R. P. Singh, "An overview on the degradability of polymer nanocomposites," Polymer Degradation and Stability, vol. 88, no. 2, pp. 234-250, 2005.

[3] E. Reynaud, T. Jouen, C. Gauthier, G. Vigier, and J. Varlet, "Nanofillers in polymeric matrix: A Study On Silica Reinforced PA6," Polymer, vol. 42, no. 21, pp. 8759-8768, 2001.

[4] F. Yang, Y. Ou, and Z. Yu, "Polyamide 6/silica nanocomposites prepared by in situ polymerization," Journal of Applied Polymer Science, vol. 69, no. 2, pp. 355-361, 1998.

[5] P. M. Ajayan, O. Stephan, C. Colliex, and D. Trauth, "Aligned carbon nanotube arrays formed by cutting a polymer resin-nanotube composite," Science, vol. 265, no. 5176, pp. 1212-1214, 1994.

[6] J. Sandler, M. S. P. Shaffer, T. Prasse, W. Bauhofer, K. Schulte, and A. H. Windle, "Development of a dispersion process for carbon nanotubes in an epoxy matrix and the resulting electrical properties," Polymer, vol. 40, no. 21, pp. 5967-5971, 1999.

[7] C. Li and T.-W. Chou, "Electrical conductivities of composites with aligned carbon nanotubes," Journal of Nanoscience and Nanotechnology, vol. 9, no. 4, pp. 2518-2524, 2009.

[8] M. Liebscher, L. Tzounis, P. Pötschke, and G. Heinrich, "Influence of the viscosity ratio in PC/SAN blends filled with MWCNTs on the morphological, electrical, and melt rheological properties," Polymer (United Kingdom), vol. 54, no. 25, pp. 68016808, 2013.
[9] F. Wode, L. Tzounis, M. Kirsten et al., "Selective localization of multi-wall carbon nanotubes in homopolymer blends and a diblock copolymer. Rheological orientation studies of the final nanocomposites," Polymer, vol. 53, no. 20, pp. 4438-4447, 2012.

[10] L. Tzounis, S. Debnath, S. Rooj et al., "High performance natural rubber composites with a hierarchical reinforcement structure of carbon nanotube modified natural fibers," Materials and Design, vol. 58, pp. 1-11, 2014.

[11] P. M. Carrasco, L. Tzounis, F. J. Mompean et al., “Thermoset magnetic materials based on poly(ionic liquid)s block copolymers," Macromolecules, vol. 46, no. 5, pp. 1860-1867, 2013.

[12] L. Tzounis, M. Kirsten, F. Simon, E. Mäder, and M. Stamm, “The interphase microstructure and electrical properties of glass fibers covalently and non-covalently bonded with multiwall carbon nanotubes," Carbon, vol. 73, pp. 310-324, 2014.

[13] D. G. Papageorgiou, L. Tzounis, G. Z. Papageorgiou, D. N. Bikiaris, and K. Chrissafis, " $\beta$-Nucleated propylene-ethylene random copolymer filled with multi-walled carbon nanotubes: mechanical, thermal and rheological properties," Polymer, vol. 55, no. 16, pp. 3758-3769, 2014.

[14] M. Du, B. Guo, and D. Jia, "Newly emerging applications of halloysite nanotubes: a review," Polymer International, vol. 59, no. 5, pp. 574-582, 2010.

[15] E. Joussein, S. Petit, J. Churchman, B. Theng, D. Righi, and B. Delvaux, "Halloysite clay minerals-a review," Clay Minerals, vol. 40, no. 4, pp. 383-426, 2005.

[16] S. Sinha Ray and M. Okamoto, "Polymer/layered silicate nanocomposites: a review from preparation to processing," Progress in Polymer Science, vol. 28, no. 11, pp. 1539-1641, 2003.

[17] E. P. Giannelis, "Polymer layered silicate nanocomposites," Advanced Materials, vol. 8, no. 1, pp. 29-35, 1996.

[18] E. P. Giannelis, "Polymer-layered silicate nanocomposites: synthesis, properties and applications," Applied Organometallic Chemistry, vol. 12, no. 10-11, pp. 675-680, 1998.

[19] S. H. Anastasiadis, K. Chrissopoulou, and B. Frick, "Structure and dynamics in polymer/layered silicate nanocomposites," Materials Science and Engineering: B, vol. 152, no. 1-3, pp. 3339, 2008.

[20] K. Chrissopoulou, I. Altintzi, S. H. Anastasiadis et al., "Controlling the miscibility of polyethylene/layered silicate nanocomposites by altering the polymer/surface interactions," Polymer, vol. 46, no. 26, pp. 12440-12451, 2005.

[21] P. A. Wheeler, J. Wang, J. Baker, and L. J. Mathias, "Synthesis and characterization of covalently functionalized laponite clay," Chemistry of Materials, vol. 17, no. 11, pp. 3012-3018, 2005.

[22] N. N. Herrera, J.-M. Letoffe, J.-P. Reymond, and E. BourgeatLami, "Silylation of laponite clay particles with monofunctional and trifunctional vinyl alkoxysilanes," Journal of Materials Chemistry, vol. 15, no. 8, pp. 863-871, 2005.

[23] K. A. Carrado, L. Xu, R. Csencsits, and J. V. Muntean, "Use of organo- and alkoxysilanes in the synthesis of grafted and pristine clays," Chemistry of Materials, vol. 13, no. 10, pp. 37663773, 2001.

[24] J. J. Tunney and C. Detellier, "Interlamellar covalent grafting of organic units on kaolinite," Chemistry of Materials, vol. 5, no. 6, pp. 747-748, 1993.

[25] L. Mercier and C. Detellier, "Preparation, characterization, and applications as heavy metals sorbents of covalently grafted thiol functionalities on the interlamellar surface of montmorillonite," Environmental Science \& Technology, vol. 29, no. 5, pp. 13181323, 1995. 
[26] M. Du, B. Guo, Y. Lei, M. Liu, and D. Jia, "Carboxylated butadiene-styrene rubber/halloysite nanotube nanocomposites: interfacial interaction and performance," Polymer, vol. 49, no. 22, pp. 4871-4876, 2008.

[27] S. R. Levis and P. B. Deasy, "Characterisation of halloysite for use as a microtubular drug delivery system," International Journal of Pharmaceutics, vol. 243, no. 1-2, pp. 125-134, 2002.

[28] S. Rooj, A. Das, V. Thakur, R. N. Mahaling, A. K. Bhowmick, and G. Heinrich, "Preparation and properties of natural nanocomposites based on natural rubber and naturally occurring halloysite nanotubes," Materials and Design, vol. 31, no. 4, pp. 21512156, 2010.

[29] T. F. Bates, F. A. Hildebrand, and A. Swineford, "Morphology and structure of endellite and halloysite," American Mineralogist, vol. 35, no. 7-8, pp. 463-484, 1950.

[30] M. Du, B. Guo, and D. Jia, “Thermal stability and flame retardant effects of halloysite nanotubes on poly(propylene)," European Polymer Journal, vol. 42, no. 6, pp. 1362-1369, 2006.

[31] Y. Ye, H. Chen, J. Wu, and L. Ye, "High impact strength epoxy nanocomposites with natural nanotubes," Polymer, vol. 48, no. 21, pp. 6426-6433, 2007.

[32] N.-Y. Ning, Q.-J. Yin, F. Luo, Q. Zhang, R. Du, and Q. Fu, "Crystallization behavior and mechanical properties of polypropylene/halloysite composites," Polymer, vol. 48, no. 25, pp. 7374-7384, 2007.

[33] B. T. Poh and C. C. Ng, "Effect of silane coupling agents on the mooney scorch time of silica-filled natural rubber compound," European Polymer Journal, vol. 34, no. 7, pp. 975-979, 1998.

[34] D. C. O. Marney, L. J. Russell, D. Y. Wu et al., "The suitability of halloysite nanotubes as a fire retardant for nylon 6," Polymer Degradation and Stability, vol. 93, no. 10, pp. 1971-1978, 2008.

[35] S. C. Lao, C. Wu, T. J. Moon et al., "Flame-retardant polyamide 11 and 12 nanocomposites: thermal and flammability properties," Journal of Composite Materials, vol. 43, no. 17, pp. 1803-1818, 2009.

[36] L. Tzounis, M. Liebscher, A. Tzounis et al., "CNT-grafted glass fibers as a smart tool for epoxy cure monitoring, UV-sensing and thermal energy harvesting in model composites," RSC Advances, vol. 6, no. 60, pp. 55514-55525, 2016.

[37] P. Yuan, P. D. Southon, Z. Liu et al., "Functionalization of halloysite clay nanotubes by grafting with $\gamma$-aminopropyltriethoxysilane," Journal of Physical Chemistry C, vol. 112, no. 40, pp. 15742-15751, 2008.

[38] M. Liu, B. Guo, M. Du, Y. Lei, and D. Jia, "Natural inorganic nanotubes reinforced epoxy resin nanocomposites," Journal of Polymer Research, vol. 15, no. 3, pp. 205-212, 2008.

[39] L. Tzounis, R. Contreras-Caceres, L. Schellkopf et al., "Controlled growth of Ag nanoparticles decorated onto the surface of $\mathrm{SiO}_{2}$ spheres: a nanohybrid system with combined SERS and catalytic properties," RSC Advances, vol. 4, no. 34, pp. 1784617855, 2014.

[40] C. Kapnopoulos, E. D. Mekeridis, L. Tzounis et al., "Fully gravure printed organic photovoltaic modules: a straightforward process with a high potential for large scale production," Solar Energy Materials and Solar Cells, vol. 144, pp. 724-731, 2016.

[41] L. Tzounis, M. Liebscher, E. Mäder, P. Pötschke, M. Stamm, and S. Logothetidis, "Thermal energy harvesting for largescale applications using MWCNT-grafted glass fibers and polycarbonate-MWCNT nanocomposites," AIP Conference Proceedings, vol. 1646, pp. 138-148, 2015.
[42] K. N. Papageridis, G. Siakavelas, N. D. Charisiou et al., "Comparative study of $\mathrm{Ni}, \mathrm{Co}, \mathrm{Cu}$ supported on $\gamma$-alumina catalysts for hydrogen production via the glycerol steam reforming reaction," Fuel Processing Technology, vol. 152, pp. 156-175, 2016. 

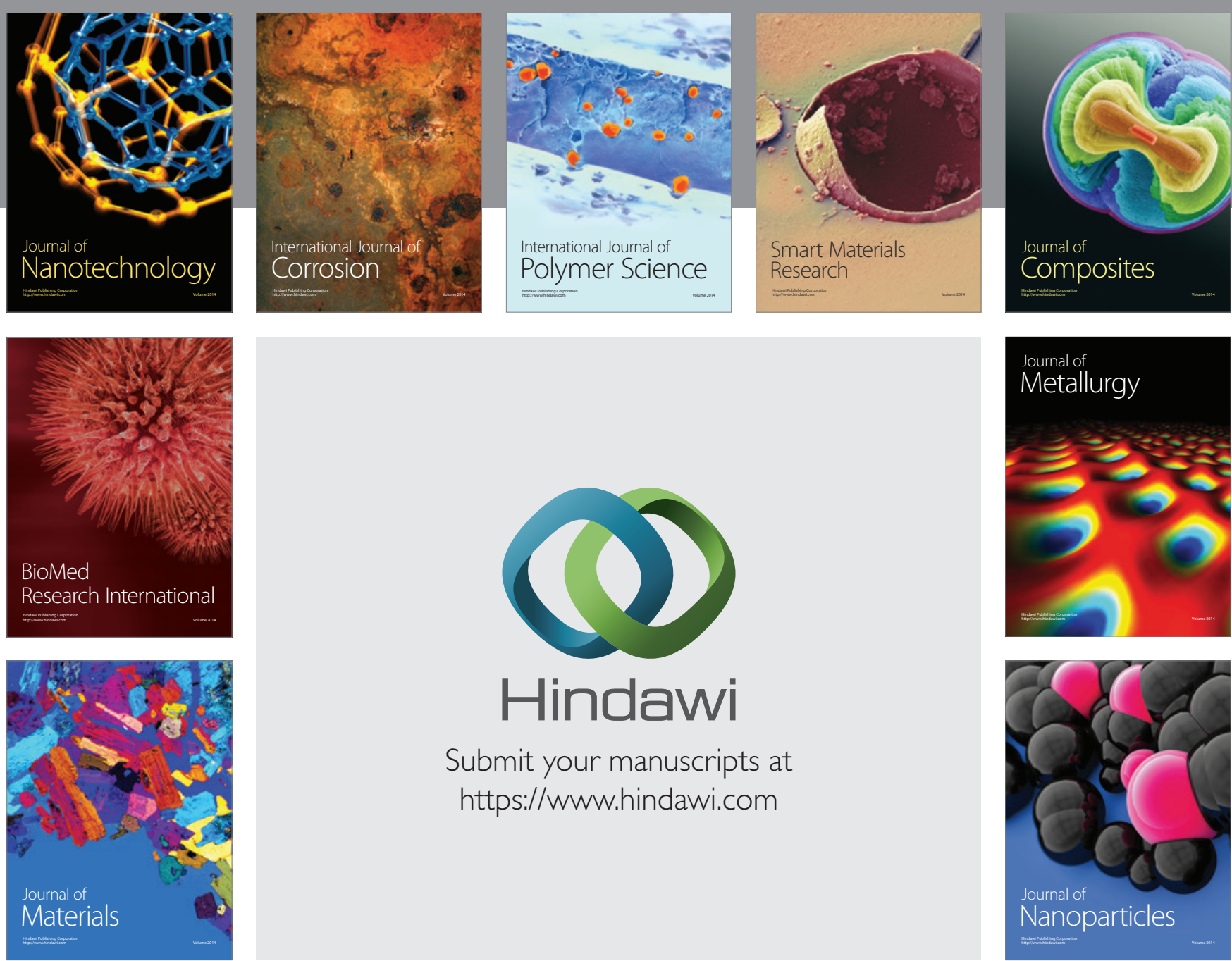

\section{Hindawi}

Submit your manuscripts at

https://www.hindawi.com

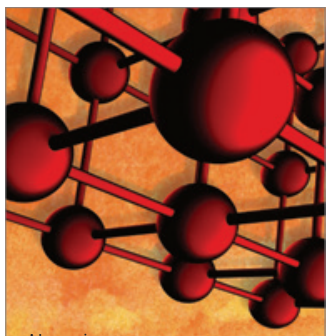

Materials Science and Engineering
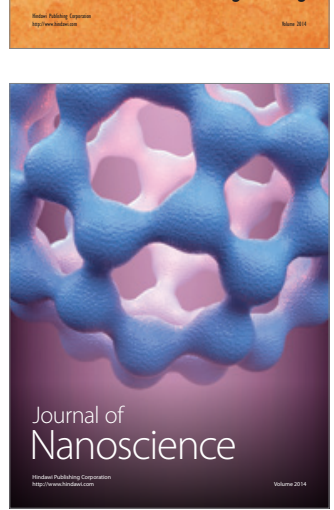
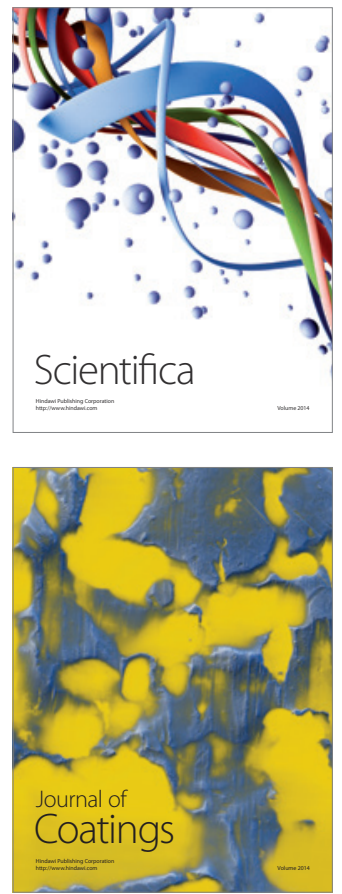
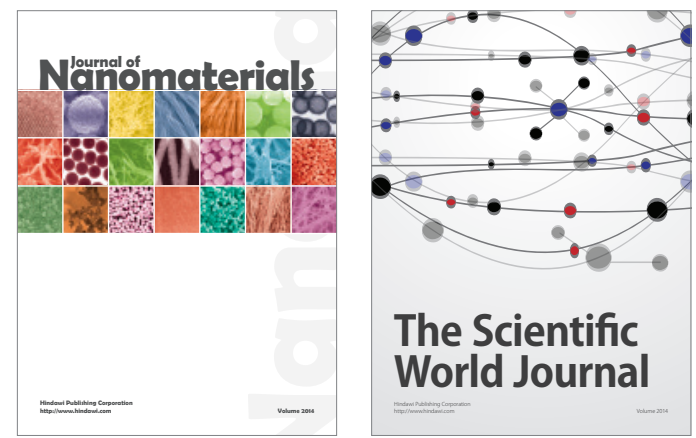

The Scientific World Journal
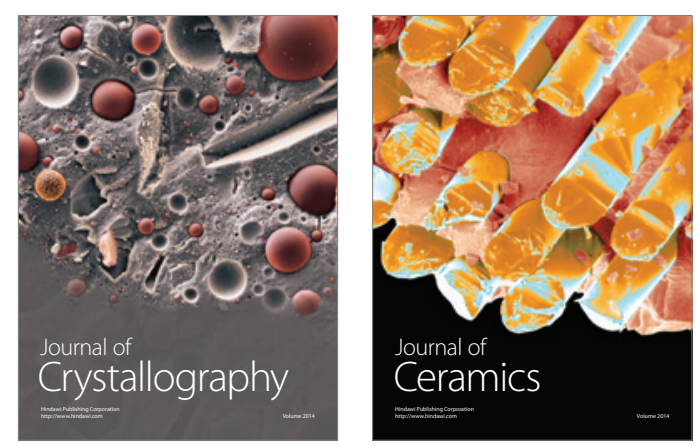
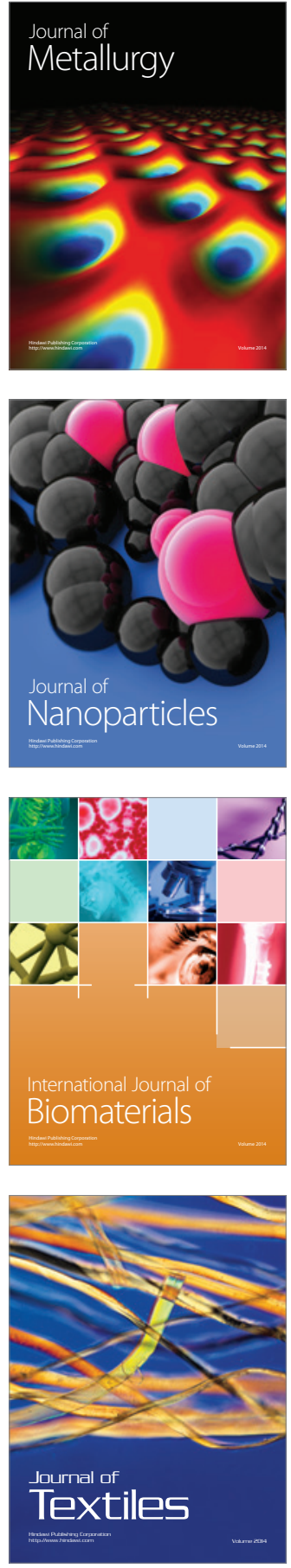